\title{
Grand challenges
}

\section{Bernhard Moser*}

Department of Infection, Immunity and Biochemistry, Cardiff University, Cardiff, UK

*Correspondence: moserb@cardiff.ac.uk

The function and eventual fate of immune cells is largely determined by factors controlling their movement through distinct parts of our body. And the molecular basis for this control is provided for by chemoattractants and cell adhesion molecules.

This importance of immune cell traffic in immunity has not always been fully appreciated. In fact, cellular immunology has traditionally concentrated on causeeffect relationships by, for instance, injecting vaccines into mice and, after a period of time, removing the spleens and lymph nodes for extraction and in vitro analysis of effector cells. Mice were viewed as black magic boxes that provided the essential inventory for allowing immune responses to occur. That immune cells need to "move" in order to achieve effector functions was clear. However, this topic was regarded as a subject of cell biology rather than immunology. This view definitely changed with the discovery of chemokines in the second half of the 1980s. Initially, chemokine studies focused on innate immunity. Consequently, progress in this new field of research was discussed at Gordon Research Conferences on Phagocytes (and related meetings), alongside with "classical" chemoattractants such as formyl-Met-LeuPhe, platelet-activating factor, leukotriene $\mathrm{B} 4$, and complement component C5a. Combining chemokines with non-peptide chemoattractants made sense since they all act via G-protein coupled, seven transmembrane domains receptors (GPCR). Unlike non-peptide chemoattractants, chemokines are related in structure, both at the level of DNA and protein and, importantly, make up the largest family of cytokines. Currently, we know the genes for $>50$ chemokines and 20 chemokine receptors. Importantly, this system is characterized by redundancies both in terms of the number of chemokines recognized by a single chemokine receptor and the number of receptors shared by individual chemokines.

The term "chemokines" stems from "chemotactic cytokines" and signifies their prototype function "chemotaxis" of immune cells, i.e., their movement along a concentration gradient of chemoattractants. By a process called "inside-out" signaling triggering of chemokine receptors induces a low-tohigh affinity shift in integrins, enabling firm adhesion of cells to integrin ligands present on other cells, such as vascular endothelial cells, or extracellular matrix. The former process is a prerequisite of immune cell extravasation and the latter process forms an integral part in immune cell chemotaxis. Both processes illustrate the intimate link between chemotaxis and cell adhesion.

Rapid progress in chemokine research revealed that not only innate immune cells but all cells of the entire immune system respond to this large class of cytokines. The control of immune cell traffic is challenged by the considerable diversity in cell types, including precursor cells in the bone marrow and thymus, mature immune cells in blood, short-lived effector T and B cells at sites of infections as well as immune surveillance cells in healthy peripheral tissues. This problem is solved by the combination of distinct chemokine receptors with adhesion receptors, yielding precise address codes for guiding individual immune cell subsets to their target tissues. Obviously, mistakes in this system can cause severe immune defects with pathophysiological consequences, including chronic infections and autoimmunity.

The field of immune cell migration poses many unsolved challenges. But chemoattractants go well beyond chemotaxis, as outlined in the below figure. In fact, the functional diversity is substantial to a degree that the writing of comprehensive review articles is a thing of the past.

The below bullet point comments illustrate some of the big challenges faced by laboratories working in this highly competitive field of research.

- Chemokine and chemokine receptor cross-talk - Physical interactions between chemokines or between chemokine/chemoattractant receptors

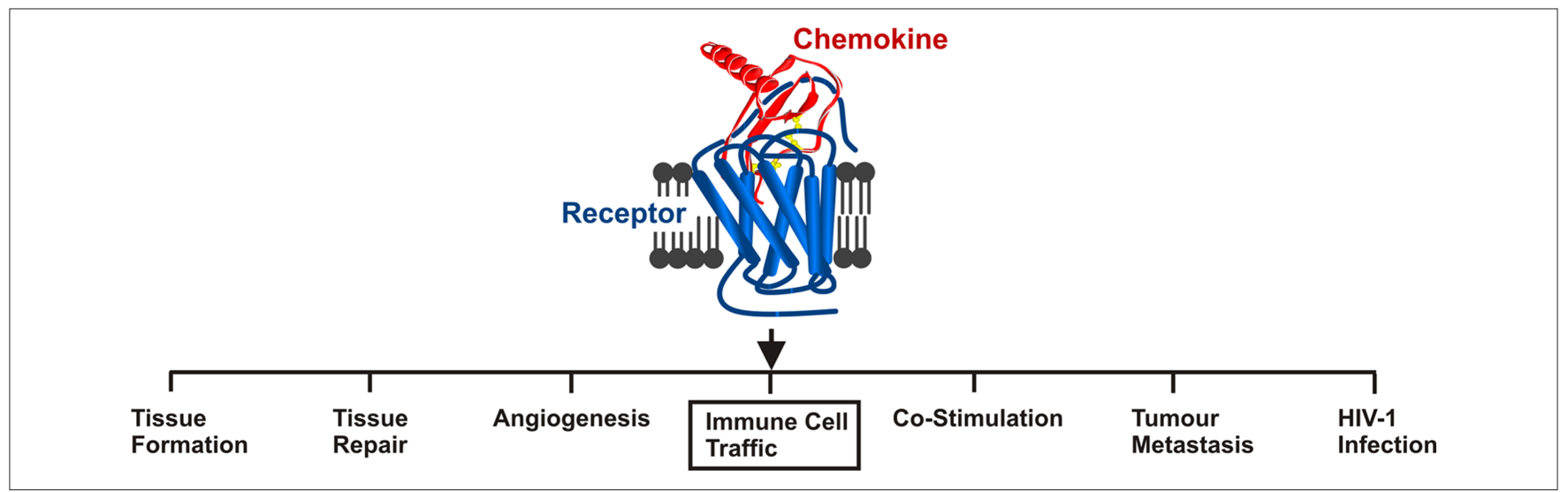


greatly influence their functions and contribute to the fine tuning of cell migration.

- Chemokine structure modifications-This topic deals with proteases and chemical modifications, adding an additional layer of complexity to the control of chemokine function and cell migration.

- In situ immune cell traffic - Life imaging of immune cell traffic has been instrumental in understanding initiation of adaptive immunity and will certainly reveal interesting novel features in immune surveillance and chronic diseases.

- Mechanisms of cell migration - The major signaling elements that are coupled to chemoattractant receptors are known. Now current research investigates how the principal mechanisms of cell migration are influenced by exterior factors (microbial products, neurotransmitters, cytokine milieu) and cell adhesion events.

- Control of innate and adaptive immunity - Current activities are based on a rich inventory of chemoattractants produced by the host, and this obvious complexity is further expanded by viral products. Investigations are trying to make "sense" by correlating individual chemoattractant systems with distinct subsets of effector/memory cells. Among other things, detailed chemokine receptor profiling will help to classify the increasing number of functional T cell subsets.

- Translational research - Only two chemokine receptor antagonists (Maraviroc from Pfizer, and ADM3100 from AnorMED) have recently gained FDA approval, but many more are in the pipelines. Of note, the new drugs do not target primarily inflammatory diseases. Major challenges faced by the pharmaceutical industry are chemokine redundancy, especially of those involved in inflammatory responses, and GPCR cross-reactivity. Promising recent compounds target cell adhesion molecules (e.g., $\alpha 4$ integrin) and innate chemoattractant receptors (e.g., C5aR/C5L2). Additional translational initiatives examine the role for inhibitors of chemokines and chemokine transporters in inflammatory diseases.

The phase of chemoattractant gene discovery is over. Now the Grand Challenges lie in the integration of the vast amount of information published in the past 22 years since work on chemokines begun in order to understand the precise role they play, together with the non-peptide chemoattractants, in immunological diseases. This demanding objective will only be reached through concerted efforts by basic biomedical and translational researchers across the globe.

Received: 18 January 2011; accepted: 19 January 2011; published online: 07 February 2011.

Citation: Moser B (2011) Grand challenges. Front. Immun. 2:1. doi: 10.3389/fimmu.2011.00001

This article was submitted to Frontiers in Chemoattractants, a specialty of Frontiers in Immunology.

Copyright (C) 2011 Moser. This is an open-access article subject to an exclusive license agreement between the authors and Frontiers Media SA, which permits unrestricted use, distribution, and reproduction in any medium, provided the original authors and source are credited. 